\section{Effect and Molecular Mechanism of Wnt/ $\beta$-Catenin Signaling Pathway on Apoptosis after Spinal Cord Injury}

Keywords: Spinal cord injury; Wnt/ $\beta$-catenin signaling pathway Apoptosis

\begin{abstract}
Secondary injury plays a more important role in the development of spinal cord injury (SCl), mainly due to apoptosis, inflammatory response, oxidative stress and other causes, especially neuronal cell apoptosis inhibits the nerve function recovery after $\mathrm{SCl}$. In recent years, the study of wht signaling after $\mathrm{SCl}$ has attracted more attention. Wnt signal pathway is divided into three kinds of signal pathway: the classic signal path (wnt/B-catenin); non-classical signal path (wnt/ JNK) and $\mathrm{Wnt}^{-\mathrm{Ca}^{2+}}$ signal path. Previous studies have shown that cell apoptosis activity is activated after inhibition of the wnt/ $\beta$-catenin signaling pathway in other systemic diseases of the body, while there is no clear description on correlation between wnt/ $\beta$-catenin signaling pathway and apoptosis after $\mathrm{SCl}$. In our previous study, we found that wnt/B-catenin signaling pathway was negatively correlated with neuronal apoptosis at different time points after $\mathrm{SCl}$. We will continue to demonstrate the effect of inhibition of activation or activation of wnt/ $\beta$-catenin signaling pathway on neuronal cell apoptosis and the effect on neurological function in vivo and in vitro experiments afte $\mathrm{SCl}$. This is the first observation of the relationship between wnt/ $\beta$ catenin signaling pathway and neuronal apoptosis after $\mathrm{SCl}$. The role of wnt/B-catenin signaling pathway in the recovery of neurologica function after $\mathrm{SCl}$ and its molecular mechanism may provide a new therapeutic target and program for treating $\mathrm{SCl}$, and provide a strong theoretical basis and experimental basis for the basic study of $\mathrm{SCl}$.
\end{abstract}

\section{Content}

Spinal cord injury (SCI) is a constant challenge in medical research and a global therapeutic problem. As a common injury, SCI often leads to irreversible physical defects that seriously influence the quality of life of patients and introduce grave burden to their family and society. SCI principally comprises two kinds of pathological processes, namely, primary and secondary injuries. Primary injury is chiefly derived from accidents, high-altitude falls, and high-energy violence. Such injuries are often irreversible and may result in uncontrollable factors in the nerve function recovery process after SCI. By contrast, secondary injury refers to the injury after SCI, involving oxidative stress, immune regulation disorder, inflammatory reaction, neuronal autophagy, and apoptotic activity. Secondary injury is an intervention and prevention issue and plays a significant role in SCI; it is also the chief target of clinical treatment after SCI $[1,2]$.

Currently, some general domestic and foreign common treatment options include (1) suitable fixation, which prevents secondary injury of the spinal cord from translocation of the injured sites; (2) reduction of spinal cord edema and secondary injury; (3) hyperbaric oxygen therapy; (4) and surgery [3,4]. However, these treatments rarely alleviate and treat SCI. Thus, a precise molecular mechanism of neural functional recovery after SCI should be developed.
Journal of Surgery

\author{
Kai Gao ${ }^{1}$ and Yingchun Zhang ${ }^{2 *}$
}

'Department of Orthopedics, Jining NO.1 People's Hospital, P.R China

${ }^{2}$ Department of Interventional Radiology, Jining No.1 People's Hospital, P.R. China

\section{*Address for Correspondence}

Yingchun Zhang, Department of Interventional Radiology, Jining No.1 People's Hospital, Jining City, P.R. China, Email: gaohaikai88@126.com

Submission: 10 April, 2018

Accepted: 23 April, 2018

Published: 30 April, 2018

Copyright: (c) 2018 Gao K, et al. This is an open access article distributed under the Creative Commons Attribution License, which permits unrestricted use, distribution, and reproduction in any medium, provided the original work is properly cited.

Apoptosis refers to the programmed cell death process regulated by cellular genes and products in the body's cells during development [5]. Apoptosis is widespread in the biosphere; this process occurs not only in normal physiological state but also in pathological conditions. Apoptosis plays important roles in embryonic development and morphogenesis, normal tissue and cell homeostasis, body defense and immune response, poison-induced disease or cell damage, aging, and tumor progression [6]. Hence, apoptosis is of great potential significance in therapy and biomedical research. During an apoptotic process, the cell nucleus shrinks and wrinkles; foam films are produced, and DNA decomposes into fragments. The apoptotic process is composed of two key components: the pro-apoptotic (e.g. caspase and Bax families) and anti-apoptotic factors (e.g. Bcl-2 family). The caspase family comprises cysteine proteases; its initial group includes caspases- $-2,-8,-9,-10$, and -11 , which are closely linked to the pro-apoptotic signaling pathway. When these enzymes are activated, they cut and activate the downstream components (caspase-3 and -6), thereby triggering apoptosis. Anti-apoptotic factors (Bcl-2 family) protect mitochondrial integrity and prevent the release of cytochrome $\mathrm{C}$ and the subsequent activation of caspase-9. Such response inhibits the apoptotic activity. The coexistence of pro- and anti-apoptotic factors maintain the organism homeostasis and cell survival $[7,8]$. Our previous study found that after SCI, the apoptosis of neuronal cells holds an important position. Following SCI, the levels of pro-apoptotic factors (caspase and Bax families) significantly increase, and those of the anti-apoptotic factors (Bcl-2 family) significantly decrease, signifying the activation of neuronal apoptosis. However, excessive neuronal apoptosis adversely affects the functional recovery after SCI [9-12]. This excess inhibits the recovery process of tissue morphology and behavior; thus, neuronal apoptosis serves as a potential target for SCI treatment. Nevertheless, the specific molecular mechanisms underlying the neuronal cell apoptosis after SCI require further exploration and research. This pursuit will provide more accurate theoretical and experimental bases for SCI treatment.

The Wnt family of glycoproteins plays an important role in the progression of central nervous system injury [13]. These glycoproteins mainly influence the proliferation, composition, and 
survival of nerve cells. The Wnt signaling pathway is mainly divided into three main signaling pathways: the classical (Wnt/ $\beta$-catenin), non-classical (Wnt/JNK) and Wnt- $\mathrm{Ca}^{2+}$ signaling pathways. When the Wnt signaling pathway is not activated, $\beta$-catenin, as an important downstream protein, functions not only as a classic cellcell adhesion adapter protein but also as a transcriptional regulatory factor. As such, $\beta$-catenin becomes marked by the APC/Axin/GSK$3 \beta$ complex and then degraded. The $\beta$-catenin protein becomes unstable in the cytoplasm, and the number of $\beta$-catenin proteins in the nucleus decreases. Ultimately, signal transduction downstream is restricted [13-15]. However, when exogenous signals are transmitted to the Wnt proteins (such as wnt-3a and $-5 \mathrm{a}$ ), these proteins combine with a cell membrane protein (Frizzled) and form protein complexes. These complexes induce LRP5/6 phosphorylation, promote GSK$3 \beta$ phosphorylation, and then inhibit $\beta$-catenin phosphorylation. $\beta$-catenin then dissociates from the APC/Axin/GSK-3 $\beta$ complexes, and the $\beta$-catenin levels in the cytoplasm are stabilized. $\beta$-catenin is transported into the nucleus and combines with LEF/TCF DNAbinding factors to serve as transcription factors. These transcription factors then activate the transcription process and regulate cell proliferation, apoptosis, and differentiation. The occurrence of this process signifies that the classic Wnt signaling pathway is activated.

Our previous study found that the canonical Wnt signaling pathway (Wnt/ $\beta$-catenin) plays an important role in SCI. After $\mathrm{SCI}$, the $\mathrm{Wnt} / \beta$-catenin signaling pathway is activated, $\beta$-catenin dissociates from the APC/Axin/GSK-3 $\beta$ complexes and receives upstream molecular signals. The protein then transports the signals to the nucleus to activate the target gene, ultimately promoting axonal regeneration and accelerating the nerve function recovery process [2,618]. Some reports on other diseases (such as acute respiratory distress syndrome and colorectal cancer) have claimed that suppressing the classical Wnt signaling pathway (Wnt/ $\beta$-catenin) would activate apoptosis and alter the normal body steady state $[19,20]$. This notion suggests that the $\mathrm{Wnt} / \beta$-catenin signaling pathway can regulate apoptotic function. Interestingly, we also preliminary verified in published articles that simvastatin inhibits neuronal apoptosis and exerts neuroprotection by activating the $\mathrm{Wnt} / \beta$-catenin signaling pathway after SCI. When the Wnt/ $\beta$-catenin signaling pathway was suppressed, the anti-apoptotic effect of simvastatin was significantly reduced after SCI. Therefore, investigating the $\mathrm{Wnt} / \beta$-catenin signaling pathway may provide a new mechanism and assist the progress of SCI treatment.

To further explore the specific molecular mechanism related to the effect of the $\mathrm{Wnt} / \beta$-catenin signaling pathway on the apoptosis of nerve cells after SCI, we accomplished a preliminary experiment and found that at different time points after SCI, the prominence of the proteins of the classical Wnt/ $\beta$-catenin signaling pathway (wnt$3 \mathrm{a}$ and $\beta$-catenin) initially increased and then decreased with time. The highest level was found at $6 \mathrm{~h}$ after SCI, whereas the lowest level existed at the third day. The expression level of the GSK-3 $\beta$ protein decreased initially and then increased again; the activity levels tended to reach the lowest peak at $6 \mathrm{~h}$ and the highest peak at 3 days after SCI. These results showed that the classic Wnt $/ \beta$-catenin signaling pathways were activated first, reached the highest activity levels at $6 \mathrm{~h}$ after SCI, and then suppressed with time with the minimum activity levels at 3 days after SCI. In addition, we observed the changes in nerve cell apoptosis levels after SCI and found that the expression levels of the proteins (caspase-3, caspase-9, and Bax) that can boost apoptosis were increased along with time and reached the highest level at 3 days after SCI then gradually decreased. On the contrary, the expression level of the Bcl-2 protein, which can suppress apoptotic activity, tended to rise first, then declined and reached the lowest level at 3 days after SCI. The consistent results showed that the level of cell apoptosis after SCI tended to increase first, with the highest level at 3 days after SCI, then decreased. The abovementioned results reveal that after SCI, the expression levels of the classic Wnt $/ \beta$ catenin signaling pathway and cell apoptosis players were negatively correlated. When the $\mathrm{Wnt} / \beta$-catenin signaling pathway is activated, nerve cell apoptosis is inhibited; when the $\mathrm{Wnt} / \beta$-catenin signaling pathways are suppressed, the nerve cell apoptosis levels significantly increased.

On the basis of the results, we propose the following points for future investigation: (1) whether a new $\mathrm{Wnt} / \beta$-catenin signaling pathway is involved in nerve cell apoptosis, (2) how the $\mathrm{Wnt} / \beta$ catenin signaling pathway controls nerve cell apoptosis after SCI, and (3) the exact role of $\mathrm{Wnt} / \beta$-catenin signaling pathway in nerve functional recovery. Furthermore, we will use different groups (inhibition or activation of $\mathrm{Wnt} / \beta$-catenin signaling pathway) to study the molecular mechanism of the $\mathrm{Wnt} / \beta$-catenin signaling pathway in the regulation of neural cell apoptosis, as well as the effects on the nerve function recovery after SCI through in vivo and in vitro experiments. We will then reveal the effects and molecular mechanism of the $\mathrm{Wnt} / \beta$-catenin signaling pathway in the regulation of neural cell apoptosis after SCI (Figure 1). This information may further promote research on the molecular mechanisms of apoptosis and the treatment of SCI, provide new molecular mechanisms and research bases, and encourage the study of the clinical treatment of SCI.

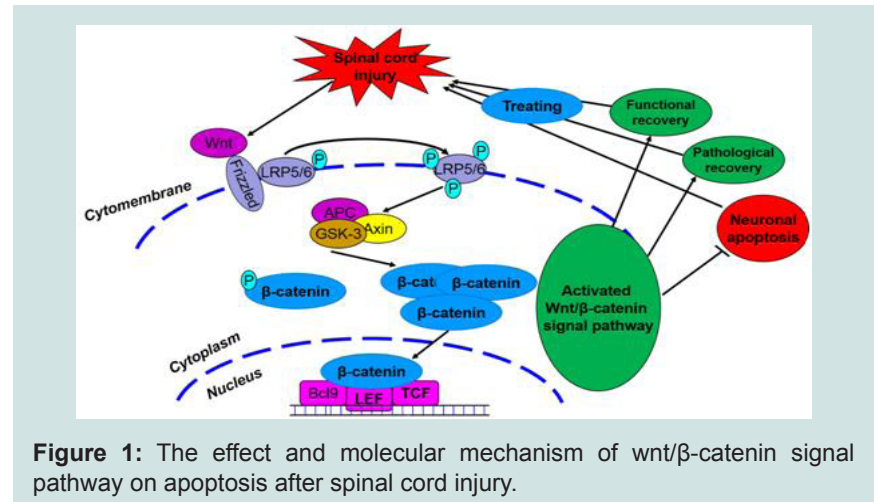

\section{References}

1. Li D, Wang G, Han D, Bi J, Li C, et al. (2016) MP resulting in autophagic cell death of microglia through zinc changes against spinal cord injury. Biomed Res Int 2016: 6090316.

2. Shen Z, Zhou Z, Gao S, Guo Y, Gao K, et al. (2017) Melatonin inhibits neural cell apoptosis and promotes locomotor recovery via activation of the $\mathrm{Wnt} / \beta$ catenin signaling pathway after spinal cord injury. Neurochem Res 42: 23362343.

3. Ludwig PE, Patil AA, Chamczuk AJ, Agrawal DK (2017) Hormonal therapy in traumatic spinal cord injury. Am J Transl Res 9: 3881-3895. 
4. Ahuja CS, Nori S, Tetreault L, Wilson J, Kwon B, et al. (2017) Traumatic spinal cord injury-repair and regeneration. Neurosurgery 80: S9-S22.

5. Taylor RC, Cullen SP, Martin SJ (2008) Apoptosis: controlled demolition at the cellular level. Nat Rev Mol Cell Biol 9: 231-241.

6. Alkabie S, Boileau AJ (2016) The role of therapeutic hypothermia after traumatic spinal cord injury--a systematic review. World Neurosurg 86: 432 449 .

7. Degterev A, Yuan J (2008) Expansion and evolution of cell death programmes. Nat Rev Mol Cell Biol 9: 378-390.

8. Lowe SW, Lin AW (2000) Apoptosis in cancer. Carcinogenesis 21: 485-495.

9. Gao K, Wang G, Wang Y, Han D, Bi J, et al. (2015) Neuroprotective effect of simvastatin via inducing the autophagy on spinal cord injury in the rat model. Biomed Res Int 2015: 260161.

10. Zhao H, Chen S, Gao K, Zhou Z, Wang C, et al. (2017) Resveratrol protects against spinal cord injury by activating autophagy and inhibiting apoptosis mediated by the SIRT1/AMPK signaling pathway. Neuroscience 348: 241 251.

11. Wang C, Liu C, Gao K, Zhao H, Zhou Z, et al. (2016) Metformin preconditioning provide neuroprotection through enhancement of autophagy and suppression of inflammation and apoptosis after spinal cord injury. Biochem Biophys Res Commun 477: 534-540

12. Zhou Z, Chen S, Zhao H, Wang C, Gao K, et al. (2016) Probucol inhibits neural cell apoptosis via inhibition of $\mathrm{mTOR}$ signaling pathway after spinal cord injury. Neuroscience 329: 193-200.
13. Gonzalez-Fernandez C, Fernandez-Martos CM, Shields SD, Arenas E, Rodríguez FJ (2014) Wnts are expressed in the spinal cord of adult mice and are differentially induced after injury. J Neurotrauma 31: 565-581.

14. Hollis ER $2^{\text {nd }}$, Zou $Y$ (2012) Expression of the Wnt signaling system in central nervous system axon guidance and regeneration. Front Mol Neurosci 5: 5.

15. Fernandez-Martos CM, Gonzalez-Fernandez C, Gonzalez P, Maqueda A, Arenas E, et al. (2011) Differential expression of Wnts after spinal cord contusion injury in adult rats. PLoS One 6: e27000.

16. Gao K, Shen Z, Yuan Y, Han D, Song C, et al. (2016) Simvastatin inhibits neural cell apoptosis and promotes locomotor recovery via activation of Wnt/ $\beta$-catenin signaling pathway after spinal cord injury. J Neurochem 138 : 139-149.

17. Gao K, Wang YS, Yuan YJ, Wan ZH, Yao TC, et al. (2015) Neuroprotective effect of rapamycin on spinal cord injury via activation of the Wnt/ $\beta$-catenin signaling pathway. Neural Regen Res 10: 951-957.

18. Lu GB, Niu FW, Zhang YC, Du L, Liang ZY, et al. (2016) Methylprednisolone promotes recovery of neurological function after spinal cord injury: association with Wnt/ß-catenin signaling pathway activation 11: 1816-1823.

19. Wang X, Xiao Y, Mou Y, Zhao Y, Blankesteijn WM, et al. (2002) A role for the beta-catenin/T-cell factor signaling cascade in vascular remodeling. Circ Res 90: 340-347.

20. Bilir B, Kucuk O, Moreno CS (2013) Wnt signaling blockage inhibits cell proliferation and migration, and induces apoptosis in triple-negative breast cancer cells. J Transl Med 11: 280.

\section{Acknowledgements}

This study was supported by the Shandong Natural Science Foundation (No. ZR2018PH024) and Jining Science and Technology Development Plan Project (No. The Zico word [2016]56-3). We thank the other researchers for the valuable technical assistance in this work. 